\title{
Diabetes mellitus due to agenesis of the dorsal pancreas in a patient with heterotaxy syndrome
}

\author{
Jo Eun Jung, $M D^{1}$, \\ Jin Ho Hur, MD', \\ Mo Kyung Jung, MD', \\ Ahreum Kwon, MD', \\ Hyun Wook Chae, MD', \\ Duk Hee Kim, MD, PhD', \\ Ho-Seong Kim, MD, PhD ${ }^{1}$
}

'Department of Pediatrics, Severance Children's Hospital, Endocrine Research Institute, Yonsei University College of Medicine, Seoul, 'Department of Pediatrics, Sowha Children's Hospital, Seoul, Korea

Received: 20 September, 2016

Revised: 11 October, 2016

Accepted: 26 October, 2016

Address for correspondence:

Ho-Seong Kim, MD, PhD

Department of Pediatrics, Severance Children's Hospital, Endocrine Research Institute, Yonsei University College of Medicine, 50-1 Yonsei-ro, Seodaemun-gu, Seoul 03722, Korea

Tel: +82-2-2228-2069

Fax: +82-2-393-9118

E-mail:kimho@yuhs.ac

https://orcid.org/0000-0003-1135099X

\begin{abstract}
Heterotaxy syndrome (HS) is a congenital disorder resulting from an abnormal arrangement of visceral organs across the normal left-right axis in the embryonic period. HS is usually associated with multiple anomalies, including defects of the major cardiovascular system and the extracardiovascular system such as intestinal malrotation, abnormal lung lobulation, bronchus anomalies, and pancreatic dysplasia. Although pancreatic dysplasia is occasionally accompanied with HS, the occurrence of diabetes mellitus (DM) due to pancreatic dysplasia in HS is rarely reported. We here report a case involving 13-year-old girl with DM caused by agenesis of the dorsal pancreas and HS diagnosed on the basis of the presence of a double-outlet right ventricle with bilateral pulmonary stenosis and intestinal malrotation with duodenal cyst. Timely diagnosis and treatment with insulin improved glycemic control.
\end{abstract}

Keywords: Diabetes mellitus, Dorsal pancreas agenesis, Heterotaxy syndrome

\section{Introduction}

Heterotaxy syndrome (HS) is a relatively rare syndrome with an incidence of 1 in 6,000 to 20,000 live births ${ }^{1)}$. The etiology of HS has been known to be the developmental failure of normal left-to-right asymmetry during the embryonic period ${ }^{2}$. It is associated with multiple anomalies, involving both cardiovascular and extracardiovascular systems, such as the gastrointestinal, hepatospelenic, neurologic, renal, and skeletal systems ${ }^{1)}$. Pancreatic dysplasia may be included in the various accompanying defects in $\mathrm{HS}^{3)}$. Although pancreatic dysplasia including agenesis or hypoplasia may be related to the occurrence of diabetes mellitus (DM), DM caused by agenesis of the dorsal pancreas in HS is rarely reported. Patients with agenesis of the dorsal pancreas have been reported to exhibit reduced islet cell mass, reduced glycogen synthesis and glycogenolysis, and increased hepatic insulin clearance, thereby resulting in the development of glucose intolerance and finally $\mathrm{DM}^{4}$. We present a unique case of HS with coexisting DM. To our knowledge, this is the first case report on a HS patient with DM due to agenesis of the dorsal pancreas.

\section{Case report}

A 13-year-old girl was referred to the pediatric endocrinology clinic for hyperglycemia (randomly checked blood glucose, $409 \mathrm{mg} / \mathrm{dL}$ and glycosylated hemoglobin [hemoglobin Alc, $\mathrm{HbAlc}], 19.0 \%$ ) with chief complaints included polyuria, polydipsia for 3 months, and weight loss (2 kg in 1 month). She did not report fever, nausea, vomiting, or abdominal pain. She was born at intrauterine period 37 weeks, with $2.02 \mathrm{~kg}$ of birth weight by Caesarean section delivery and followed by the pediatric cardiology department for double-outlet-right ventricle (DORV) with stenosis in both pulmonary arteries since birth. A review of her family history found type $2 \mathrm{DM}$ in her paternal grandmother. Her height and weight were $154.5 \mathrm{~cm}$ (10th- 
25th percentile) and $42.1 \mathrm{~kg}$ (10th-25th percentile), respectively, and her body mass index was $17.9 \mathrm{~kg} / \mathrm{m}^{2}$ (10th-25th percentile), which was below average. Abdominal tenderness or organomegaly was not found on physical examination.

An oral glucose tolerance test (OGTT) was performed, and the results were high serum glucose levels $(250 \mathrm{mg} / \mathrm{dL}$ at baseline, $506 \mathrm{mg} / \mathrm{dL}$ at 2 hours after oral glucose $75 \mathrm{~g}$ load) and not low C-peptide levels $(1.95 \mathrm{ng} / \mathrm{mL}$ at baseline, $4.60 \mathrm{ng} / \mathrm{mL}$ at 2 hours after oral glucose load). The titers of autoantibodies (islet cell antibody, antiglutamic acid decarboxylase antibody, and anti-insulin antibody) were negative. Aspartate aminotransferase (AST) and alanine aminotransaminase (ALT) levels were elevated (152 IU/L and $296 \mathrm{IU} / \mathrm{L}$, respectively), whereas the results of other laboratory tests, including complete blood cell count, and blood chemistry (pancreatic function, electrolyte, serum osmolality, urine osmolality, lipid profile, and thyroid function test) were unremarkable. Abdominal sonography was performed to evaluate the cause of AST and ALT elevation, which revealed intestinal malrotation and partial agenesis of the dorsal pancreas in addition to nonspecific finding on liver. Abdominal computed tomography confirmed intestinal malrotation with a duodenal cyst and partial agenesis of the dorsal pancreas (Fig. 1). The combination of DORV with pulmonary artery stenosis and multiple extracardiovascular anomalies related to abnormal left-to-right axis asymmetry led to the diagnosis of HS and DM. In addition, elevated AST and ALT changed to normal after 1 week, with conclusion of finally recovered acute hepatitis.

An oral hypoglycemic agent was prescribed, which was continued for 6 months. However, the patient's hyperglycemia was poorly controlled, and her $\mathrm{HbAlc}$ level remained elevated (around 17\%-19\%) despite excellent drug compliance. In addition, her C-peptide level decreased gradually for 6 months (preprandial level, from $1.95 \mathrm{ng} / \mathrm{mL}$ to $1.40 \mathrm{ng} / \mathrm{mL}$ and to 1.67 $\mathrm{ng} / \mathrm{mL}$; postprandial level, from $4.60 \mathrm{ng} / \mathrm{mL}$ to $2.52 \mathrm{ng} / \mathrm{mL}$ and to $1.41 \mathrm{ng} / \mathrm{mL}$ at the 3 -month period and the 6 -month period). Insulin therapy was initiated, after which hyperglycemia began to be controlled. Her HbAlc level also started to decrease (to $15.0 \%$ after 3 months of insulin treatment) (Fig. 2). She now receives insulin injection 4 times daily (long-acting insulin once every morning and 3 injections of a preprandial rapid-acting insulin analogue and the dose of insulin was 20 unit of longacting insulin once for every morning and 10 units three times a day for every preprandial rapid-acting insulin, total 1 unit per $\mathrm{kg}$ ) without an oral hypoglycemia agent. However, HbAlc was still checked high because her compliance to inject insulin daily was poor. Thus we educated her again, and we expected that $\mathrm{HbAlc}$ decreases gradually as times go by. We intended to check her serum glucose levels and $\mathrm{HbAlc}$ regularly.

\section{Discussion}

HS is a rare disease with multiple congenital anomalies originating from failure of the normal left-to-right asymmetry during the development of the embryo ${ }^{1)}$. Various congenital disorders, such as congenital heart anomalies, gastrointestinal anomalies, liver dysfunction, pancreas hypoplasia, adrenal mass, genitourinary tract anomalies, neural defects, skeletal defects, and respiratory tract defects, may accompany HS. In this case, a patient with known DORV with bilateral pulmonary artery hypoplasia was incidentally found to have intestinal malrotation

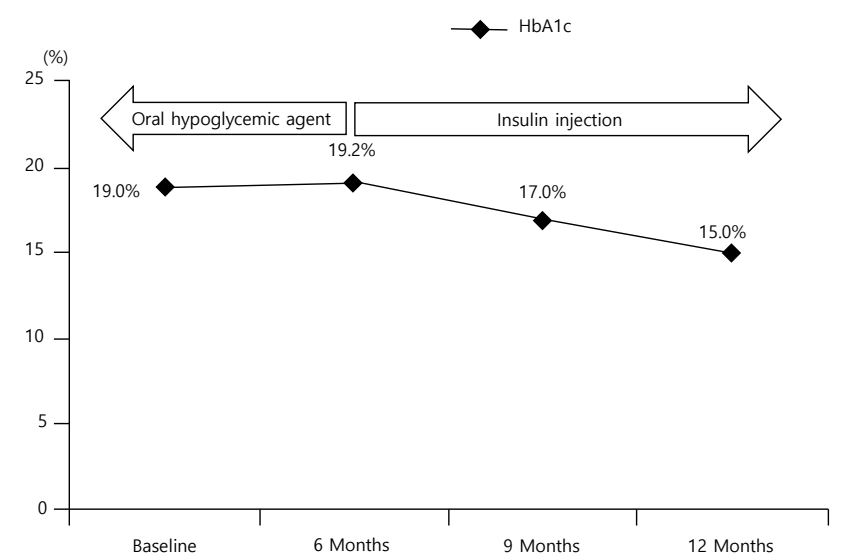

Fig. 2. Progression of hemoglobin A1c (HbA1c) in the patient.
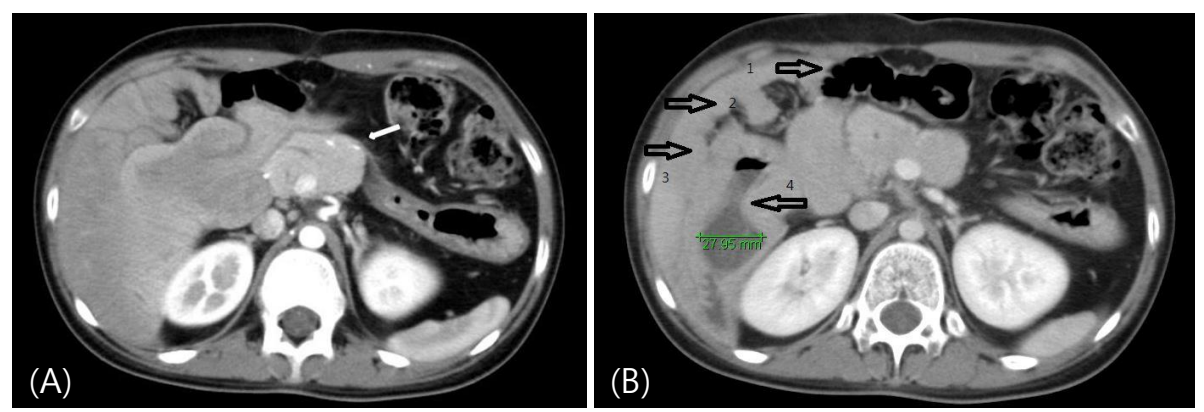

Fig. 1. (A) Axial computed tomography image showing a partial agenesis of pancreas with non-visible tail of the pancreas. Visible portion of the pancreas is just head of the pancreas (white arrow). (B) Axial computed tomography image showing intestinal malrotation of right sided small bowel and left sided large bowel and about 27.95-mm duplication cyst from duodenum with intussusception (arrow 1, left sided large bowel; arrow 2-3, right sided small bowel; arrow 4, duplication cyst from duodenum with intussuception) 
and partial pancreatic agenesis with duodenal cyst. Accordingly, we diagnosed her condition as HS considering the major cardiac anomalies accompanying extracardiovascular anomalies such as agenesis of the dorsal pancreas and intestinal malrotation.

The pancreas develops from the dorsal and ventral buds, which appear from the caudal region of the embryonic foregut. Two buds unite during the development and rotation of the embryo. Thereafter, the portion of the dorsal bud mainly forms the ventrosuperior part of the head, isthmus, tail, and body of the pancreas. The ventral bud forms the uncinate process and the posteroinferior part of the head ${ }^{5,6)}$. Agenesis of the dorsal pancreas may stem from an abnormal arrangement of organs across the left-to-right axis. Thus, agenesis of the dorsal pancreas is also one of the accompanying anomalies of HS. Although an annular pancreas, truncated pancreas, and pancreatic malrotation associated with HS have been reported $^{7-9)}$, the incidence of pancreatic abnormalities in HS has not been exactly studied. Pancreatic abnormalities in HS can lead to increased incidence of pancreatitis, DM, and obstructive jaundice $^{10)}$.

Complete agenesis of the pancreas is a rare cause of neonatal $\mathrm{DM}$, which is associated with malabsorption, severe intrauterine growth retardation, and usually fatal outcomes ${ }^{11-13}$. Partial agenesis of the ventral pancreas is described as a very rare congenital anomaly ${ }^{14)}$, and its coincidence with DM has not been reported. Partial agenesis of the dorsal pancreas can be a cause of DM. It has been reported that $53 \%$ of patients with agenesis of the dorsal pancreas present with hyperglycemia, and approximately half of them require insulin treatment ${ }^{5,6,15,16)}$. It has been reported that the islet concentration in the tail is significantly greater than that in the head and body ${ }^{17)}$. Therefore, agenesis of the dorsal pancreas including the tail may cause DM. In a family with agenesis of the dorsal pancreas, intravenous glucose tolerance test showed impaired response of first-phase insulin secretion in the diabetic mother ${ }^{4)}$. In addition, reduced glycogen synthesis and breakdown, and increased gluconeogenesis and hepatic insulin clearance were demonstrated in the diabetic mother and her son with glucose tolerance ${ }^{4}$. These findings suggest that reduced islet cell mass and defects in hepatic glycogen metabolism in patients with agenesis of the dorsal pancreas might contribute to the development of DM. The patient in this study finally needed multiple daily injections of insulin to control her hyperglycemia.

The case of DM with HS had not been reported. Thus, we can predict development and progress of DM with HS through our case review and compared to patients of pancreas partial agenesis. In conclusion, we report the first case of DM due to agenesis of the dorsal pancreas in a patient with HS. Although the coincidence of agenesis of the dorsal pancreas with HS is an extremely rare anomaly, the necessity of measurements of serum glucose, C-peptide or OGTT should be considered during follow-up since the time of the initial diagnosis, in the case of HS with pancreas anomaly.

\section{Conflict of interest}

No potential conflict of interest relevant to this article was reported.

\section{References}

1. Britz-Cunningham SH, Shah MM, Zuppan CW, Fletcher WH. Mutations of the Connexin 43 gap-junction gene in patients with heart malformations and defects of laterality. N Engl J Med 1995;332:1323-9.

2. Shiraishi I, Ichikawa H. Human heterotaxy syndrome from molecular genetics to clinical features, management, and prognosis - . Circ J 2012;76:2066-75.

3. Williams GD, Feng A. Heterotaxy syndrome: implications for anesthesia management. J Cardiothorac Vasc Anesth 2010;24:834-44.

4. Stingl H, Schnedl WJ, Krssak M, Bernroider E, Bischof MG, Lahousen T, et al. Reduction of hepatic glycogen synthesis and breakdown in patients with agenesis of the dorsal pancreas. J Clin Endocrinol Metab 2002;87:4678-85.

5. Schnedl WJ, Piswanger-Soelkner C, Wallner SJ, Reittner P, Krause R, Lipp RW, et al. Agenesis of the dorsal pancreas and associated diseases. Dig Dis Sci 2009;54:481-7.

6. Joo YE, Kang HC, Kim HS, Choi SK, Rew JS, Chung MY, et al. Agenesis of the dorsal pancreas: a case report and review of the literature. Korean J Intern Med 2006;21:236-9.

7. Kapa S, Gleeson FC, Vege SS. Dorsal pancreas agenesis and polysplenia/heterotaxy syndrome: a novel association with aortic coarctation and a review of the literature. JOP 2007;8:433-7.

8. Adeyemi SD. Combination of annular pancreas and partial situs inversus: a multiple organ malrotation syndrome associated with duodenal obstruction. J Pediatr Surg 1988;23:188-91.

9. Ito H, Ohgi S, Kanno T, Ishibashi T. Heterotaxy syndrome with pancreatic malrotation: CT features. Abdom Imaging 2003;28:856-8.

10. Mishra S. Cardiac and non-cardiac abnormalities in heterotaxy syndrome. Indian J Pediatr 2015;82:1135-46.

11. Barbarini DS, Haslinger V, Schmidt K, Patch AM, Müller G, Simma B. Neonatal diabetes mellitus due to pancreas agenesis: a new case report and review of the literature. Pediatr Diabetes 2009;10:487-91.

12. Ashraf A, Abdullatif H, Hardin W, Moates JM. Unusual case of neonatal diabetes mellitus due to congenital pancreas agenesis. Pediatr Diabetes 2005;6:239-43.

13. Chen R, Hussain K, Al-Ali M, Dattani MT, Hindmarsh P, Jones PM, et al. Neonatal and late-onset diabetes mellitus caused by failure of pancreatic development: report of 4 more cases and a review of the literature. Pediatrics 2008;121:e1541-7.

14. Kamisawa T, Tu Y, Egawa N, Ishiwata J, Okamoto A. Hypoplasia of ventral pancreas shows a threadlike ventral 
pancreatic duct. Pancreas 1999;18:214-5.

15. Lång K, Lasson A, Müller MF, Thorlacius H, Toth E, Olsson R. Dorsal agenesis of the pancreas - a rare cause of abdominal pain and insulin-dependent diabetes. Acta Radiol 2012;53:2-4.
16. Shimodaira M, Kumagai N, Sorimachi E, Hara M, Honda K. Agenesis of the dorsal pancreas: a rare cause of diabetes. Intern Emerg Med 2012;7:83-4.

17. Wittingen J, Frey CF. Islet concentration in the head, body, tail and uncinate process of the pancreas. Ann Surg 1974;179:412-4. 